\title{
Adaptive Morphological Feature Extraction and Support Vector Regressive Classification for Bearing Fault Diagnosis
}

\author{
Jun Shuai, ${ }^{1,2}$ Changqing Shen, ${ }^{1}$ and Zhongkui Zhu ${ }^{1}$ \\ ${ }^{1}$ School of Urban Rail Transportation, Soochow University, Suzhou 215131, China \\ ${ }^{2}$ School of Mechanical and Electric Engineering, Soochow University, Suzhou 215131, China \\ Correspondence should be addressed to Changqing Shen; cqshen@suda.edu.cn
}

Received 2 December 2016; Revised 18 February 2017; Accepted 23 February 2017; Published 13 March 2017

Academic Editor: Pavan K. Kankar

Copyright (c) 2017 Jun Shuai et al. This is an open access article distributed under the Creative Commons Attribution License, which permits unrestricted use, distribution, and reproduction in any medium, provided the original work is properly cited.

\begin{abstract}
Numerous studies on fault diagnosis have been conducted in recent years because the timely and correct detection of machine fault effectively minimizes the damage resulting in the unexpected breakdown of machineries. The mathematical morphological analysis has been performed to denoise raw signal. However, the improper choice of the length of the structure element (SE) will substantially influence the effectiveness of fault feature extraction. Moreover, the classification of fault type is a significant step in intelligent fault diagnosis, and many techniques have already been developed, such as support vector machine (SVM). This study proposes an intelligent fault diagnosis strategy that combines the extraction of morphological feature and support vector regression (SVR) classifier. The vibration signal is first processed using various scales of morphological analysis, where the length of SE is determined adaptively. Thereafter, nine statistical features are extracted from the processed signal. Lastly, an SVR classifier is used to identify the health condition of the machinery. The effectiveness of the proposed scheme is validated using the data set from a bearing test rig. Results show the high accuracy of the proposed method despite the influence of noise.
\end{abstract}

\section{Introduction}

Given the rapid development of industrial technology, numerous multifunctional machineries have been employed to replace humans, particularly in dangerous environments. High production efficiency considerably relies on the continuous operation of machineries. However, unexpected machine breakdowns often occur due to mechanical faults, thereby resulting in huge economic losses and even threatening human safety. Researchers have determined that most machine failures are caused by faults in key components, such as bearings and gearboxes $[1,2]$. Hence, the demand to inspect the health condition of these crucial components is increasing, and an efficient and intelligent machine fault diagnosis method should be developed to improve the reliability, safety, and effectiveness of operating systems $[3,4]$.

Extensive studies have been conducted in recent years to improve the effectiveness of fault diagnosis methods. Signal processing- and pattern recognition-based methods are the two main categories of data-driven techniques. Fault signal processing refers to the extraction of fault-related features from raw signals, in which faulty impulses are whelmed with noise. Consequently, a few related theories or methods have been developed. Ben Ali et al. [5] conducted a mathematical analysis to select the most significant intrinsic mode functions after an empirical mode decomposition of the bearing fault signal and evaluated the bearing condition and defect severity. Seshadrinath et al. [6] introduced complex wavelets in multiple fault diagnoses and proved the applicability of this scheme for industrial drives under variable frequencies and load conditions. Cong et al. [7] proposed the slip matrix construction method based on singular value decomposition. Given that the bearing runs from normal state to failure, the initial fault signal component can be selected from the entire life vibration data, thereby achieving an excellent performance in fault detection. Tiwari et al. [8] extracted the bearing fault features based on multiscale permutation entropy, which proved to be a reliable and automated fault diagnosis approach. Wang et al. [9] used the kurtosis extracted from the signal processed by short-time 
Fourier transform to establish the kurtogram; the enhanced kurtogram was effective in detecting various bearing faults. Zhang et al. [10] improved the Hilbert-Huang transform spectrum, which was constructed with the relevant and nearly monochromatic IMFs. A substantially accurate timefrequency distribution was produced for the inspected signal.

Morphological analysis has also been extensively performed to evaluate the satisfactory performance of signal processing in noise reduction. This process is an originally developed nonlinear method that uses structure elements (SEs) to measure and extract the corresponding shape of a given image [11]. Morphological analysis is also effective in many aspects, such as surface chemistry [12]. Moreover, machine fault diagnosis extracts the fault-related impacts with specific shapes, which are obtained using the component mechanism. Therefore, morphological analysis is applicable in extracting or enhancing the fault features. Dong et al. [13] effectively identified the rotating machinery fault by using a morphological filter, which is optimized by the particle swarm optimization algorithm and nonlinear manifold learning algorithm local tangent space alignment. Rajabi et al. [14] proposed a novel approach by combining mathematical morphology and multioutput adaptive neurofuzzy inference system classifier. Li et al. [15] established a multiscale morphological filtering of the vehicle system model, which displays considerable noise reduction performance. The results demonstrated that the proposed method can extract the influential characteristics of axle box vibration signals and effectively diagnose real-time wheel flat faults. Hong et al. [16] designed a gear fault diagnosis method based on the morphological mean wavelet transform, which has a simple structure, easy realization, sensitive local extremum signal, and high denoising ability, to determine the position of the impact signal. Raj and Murali [17] introduced a new method for the selection of SEs that depends on kurtosis, which is effective and robust in bringing out the impulses from bearing fault signals. Li et al. [18] calculated the general mathematical morphology particle from the normal state to failure; the calculated index was proven to be a valuable indicator of the degradation of the bearing performance. Yu et al. [19] applied an improved morphological component analysis to separate the meshing and periodic impulse components. Several case studies validated the effectiveness of diagnosing the compound fault of gearboxes. Bhateja et al. [20] introduced a scheme that combines the wavelet analysis and morphological filtering in the ECG field. The previous study has completed a few accomplishments in processing and extracting the features of machinery fault. However, the selection of the length of SE is crucial to properly measure and extract such features. Most of them are selected based on the experience or complicated indicators, which is time consuming and inapplicable to online processing.

Expertise is necessary to implement a signal processingbased method to correctly diagnose the machine fault using the resulting signal. However, the acquired signal is often obtained from multiple sources due to the environment where the machine works; the extraction of the machine fault-related component becomes difficult as well. Consequently, after the denoising process and fault characteristic extraction, an appropriate pattern recognition method is selected to map the features of the fault type. In recent years, several machine learning algorithms, such as support vector machine (SVM), deep neural network, and clustering algorithm, have been employed for this objective [21]. Vapnik [22] proposed SVM, which is regarded as a promising tool for classification with well-defined formulation and performs well in a few sample circumstances. SVM is a binary classifier. However, the condition of the bearing often contains the inner race fault, outer race fault, ball fault, and health status. A few strategies, such as the direct acyclic graph, one against all, and one against one, can be applied to solve this problem [23]. The one against one strategy is the recommended and preferred strategy for the actual application because of its rapid training speed and satisfactory accuracy in classification. However, this strategy is a voting approach. Multiple binary classifiers should be constructed and the samples should be fed into these classifiers to obtain the vote for each class. The class with the highest votes is the output class of the related sample, thereby suffering from equal votes problem. These strategies have drawbacks and increase the computational burden. Support vector regression (SVR), which was developed from SVM, can address the problem with continuous output or target value. Hence, SVR is commonly applied for time series analysis. Gu et al. [24] developed an incremental v-SVR based on v-SVC. The case study on benchmark data sets proved that the incremental SVR learning algorithm can avoid the infeasible updating paths by converging to the optimal solution. Kazem et al. [25] proposed a stock market price predicting method based on SVR. The SVR hyperparameters were optimized using the firefly algorithm and chaos theory. The phase space dynamics were reconstructed using a delay coordinate embedding method and eventually reached high prediction accuracy. Kavousi-Fard et al. [26] used SVR to obtain the accurate electrical load estimate, which outperformed the traditional techniques. Wei et al. [27] selected the parameters of SVR that were estimated through particle filtering and applied thereafter in reliability prediction. Chen and $\mathrm{Yu}$ [28] used SVR on the basis of an unscented Kalman filter to precisely update the short-term estimation of wind speed sequence.

This study presents a novel intelligent machine fault diagnosis procedure. The signals are first preprocessed using the improved morphology analysis, which selects the SE length adaptively. Thereafter, a feature vector with nine statistical values is calculated from the processed vibration signal for each sample. SVR theory is used as basis to develop a regressive classifier to overcome the SVM problems. Lastly, one case study for bearing is conducted to verify the satisfactory performance of the proposed procedure. Moreover, SVR exhibits an improved accuracy compared with other fault diagnosis schemes.

The rest of this paper is organized as follows. Section 2 provides a brief description of the principal theory of a morphological analysis and SVR. Section 3 introduces the proposed intelligent fault diagnosis procedure. Section 4 discusses the case study that validates the performance of the proposed method. Lastly, Section 5 presents the conclusion. 


\section{Theoretical Background}

2.1. Morphological Analysis. Serra [29] first introduced morphological analysis in 1982 and used SEs to collect information or deform the shape of an image. Morphological analysis has been verified to exhibit an outstanding performance in denoising. This method functions with two basic operators as follows:

Erosion

$$
\begin{aligned}
(f \Theta g)(n) & =\min [f(n+m)-g(m)] \\
m & =0,1,2, \ldots, M-1, n=0,1,2, \ldots, N-1
\end{aligned}
$$

\section{Dilation}

$$
\begin{aligned}
(f \oplus g)(n) & =\max [f(n-m)+g(m)], \\
m & =0,1,2, \ldots, M-1, n=0,1,2, \ldots, N-1,
\end{aligned}
$$

where $f(n)$ is the original one-dimensional vibration signal, $g(m)$ is the SE, and $\Theta$ and $\oplus$ are the operators of erosion and dilation, respectively. Erosion calculation is used to suppress and smooth the positive and negative impacts, respectively. By contrast, dilation calculation is used to flatten and suppress the positive and negative impacts, respectively. Another two operators are created on the basis of two basic operators as follows:

Opening

$$
(f \circ g)(n)=(f \Theta g \oplus g)(n), \quad n=0,1,2, \ldots, N-1
$$

\section{Closing}

$$
(f \cdot g)(n)=(f \oplus g \Theta g)(n), \quad n=0,1,2, \ldots, N-1,
$$

where $\circ$ and - represent the opening and closing functions, respectively. The opening operator suppresses and preserves the positive and negative impacts, respectively. By contrast, the closing operator suppresses and preserves the negative and positive impacts, respectively.

The preceding four operators only calculate the feature information from one aspect and may lose a few geometric characteristics of the signal, which is meaningful in fault diagnosis. To detect the impulsive components, the closing and opening operators are combined to establish the difference operator, which can extract the positive and negative fault impacts.

\section{Difference}

$$
\left(f \cdot g_{n}-f \circ g\right)(n)=(f \oplus g \Theta g-f \Theta g \oplus g)(n) .
$$

The performance of morphological analysis depends on the operators and SEs; therefore, selecting an appropriate SE is significant. SEs are mainly determined by the length, height, and shape. SEs with a straight line shape have been determined to perform well [30]; hence, considerable attention should be focused to determine the length of SEs.
2.2. Support Vector Regression. SVR is a type of machine learning algorithm that uses support vector to realize the function of regression [31]. Let $\left\{x_{i}, y_{i}\right\}_{i=1}^{L}$ denote the training set, where $x_{i}$ denotes the $i$ th input feature vector and $y_{i}$ denotes the $i$ th output pattern (i.e., its label). $\varepsilon$-insensitive SVR aims to find the function $f(x)$ :

$$
f(x)=w \cdot x+b,
$$

where $w$ is the coefficient vector that is used to represent the place of function in the space and $b$ is a constant quantity. The question is to find the proper $w$ and $b$. Moreover, $\varepsilon$ is introduced when considering the error-tolerant rate; hence, the question has changed as follows:

$$
\begin{array}{ll}
\min & \frac{1}{2}\|w\|^{2} \\
\text { s.t. } & y_{i}-w \cdot x_{i}-b \leq \varepsilon \\
& w \cdot x_{i}+b-y_{i} \leq \varepsilon
\end{array}
$$

where $\varepsilon$ denotes the fitting precision. To reduce the influence of the outlier, the slack variables $\xi_{i}$ are introduced:

$$
\begin{array}{ll}
\min & \frac{1}{2}\|w\|^{2}+C \sum_{i=1}^{L}\left(\xi_{i}+\xi_{i}^{*}\right), \\
\text { s.t. } & y_{i}-w \cdot x_{i}-b \leq \varepsilon+\xi_{i}, \\
& w \cdot x_{i}+b-y_{i} \leq \varepsilon+\xi_{i}^{*} \\
& \xi_{i}, \xi_{i}^{*} \geq 0
\end{array}
$$

where $C$ is the penalty factor. $\xi_{i}$ and $\xi_{i}^{*}$ are slack variables that denote the deviation from the functional margin.

The application of the Lagrange multipliers $\alpha_{i}$ and $\alpha_{i}^{*}$ can change the formula as follows:

$$
\begin{array}{ll} 
& W\left(\alpha, \alpha^{*}\right) \\
& =-\frac{1}{2} \sum_{i, j=1}^{L}\left(\alpha_{i}-\alpha_{i}^{*}\right)\left(\alpha_{j}-\alpha_{j}^{*}\right)\left(x_{i} \cdot x_{j}\right) \\
& +\sum_{i=1}^{L}\left(\alpha_{i}-\alpha_{i}^{*}\right) y_{i}-\sum_{i=1}^{L}\left(\alpha_{i}+\alpha_{i}^{*}\right) \varepsilon \\
\text { s.t. } \quad \sum_{i=1}^{L}\left(\alpha_{i}-\alpha_{i}^{*}\right)=0, \\
\quad 0 \leq \alpha_{i}, \alpha_{i}^{*} \leq C, i=1,2, \ldots, L .
\end{array}
$$

To efficiently address the nonlinear regression question, the kernel function $K\left(x_{i}, x\right)$ is introduced and the nonlinear regression function fitting is obtained as follows:

$$
f(x)=\sum_{i=1}^{L}\left(\alpha_{i}-\alpha_{i}^{*}\right) K\left(x_{i}, x\right)+b .
$$




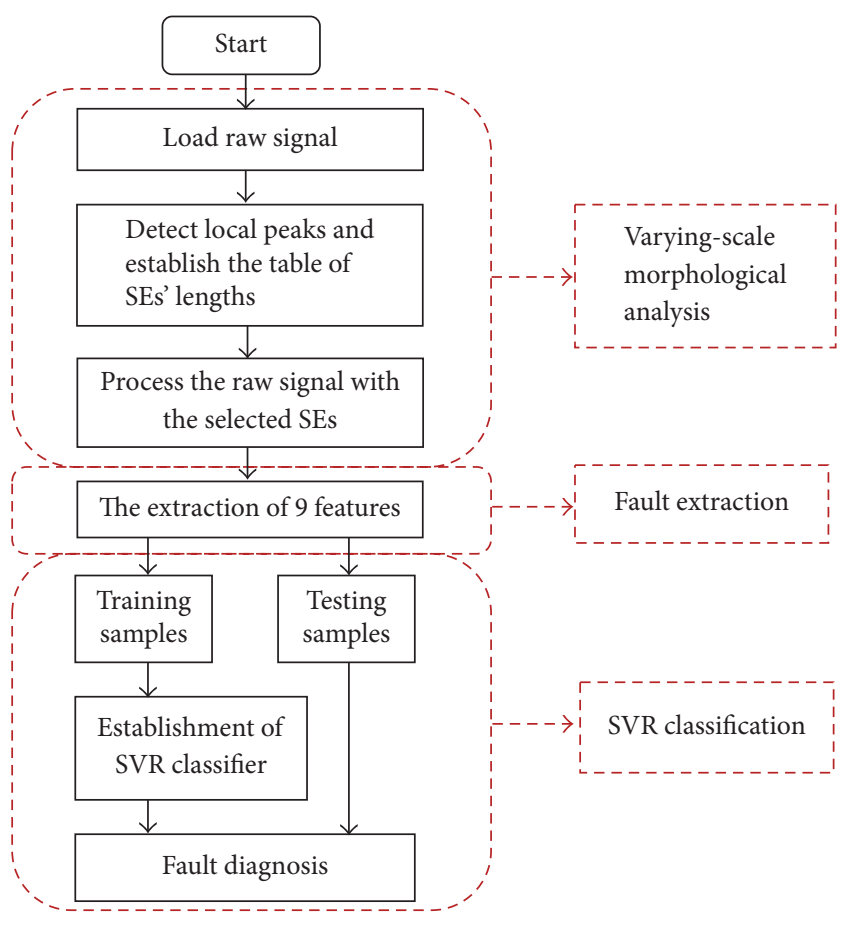

FIGURE 1: Flowchart of the proposed scheme.

\section{Proposed Fault Identification Scheme}

The proposed intelligent fault diagnosis procedure (see Figure 1) is based on the extraction of an adaptive morphological feature and support vector regressive classifier. The proposed procedure mainly comprises three steps: varyingscale morphological analysis, feature extraction, and SVR classification. The following subsections depict the details.

3.1. The Varying-Scale Morphological Analysis. Different from the traditional morphological analysis, the proposed varyingscale morphological analysis considers noise distribution. In various moments, the fault features are whelmed in varying degrees under the complicated environment. Hence, a fixed SE length may be unable to suppress the noise and properly enhance the fault feature. This study proposes a varyingscale morphological analysis to overcome the shortcomings of traditional methods. The lengths of SEs are determined dynamically using the intervals between the two adjoining local peaks, that is, by considering the dynamic effect of noise.

Hence, the SE $g(m)$ changes with time point and new SE $g_{n}(m)$ is proposed as a substitute to $g(m)$. The difference operator can be changed as follows:

Difference

$$
\begin{aligned}
(f & \left.\bullet g_{n}-f \circ g_{n}\right)(n) \\
\quad= & \left(f \oplus g_{n} \Theta g_{n}-f \Theta g_{n} \oplus g_{n}\right)(n) .
\end{aligned}
$$

Accordingly, the SE $g(m)$ in the erosion, dilation, opening, and closing transforms is replaced by the redefined SE $g_{n}(m)$.

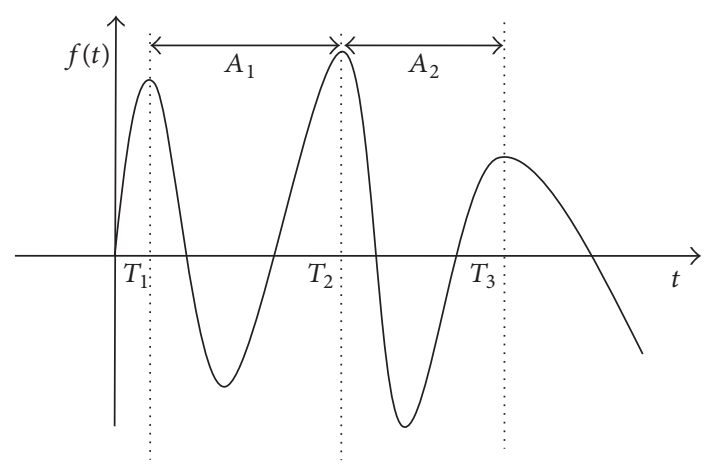

FIgUre 2: The dynamical definition of the SEs' lengths.

When the surface of a mechanical component suffers a defect during its interaction with other surfaces, a fault-related impact is generated in the captured vibration waveforms. Meanwhile, the obtained signal is polluted in the environment with additional useless impacts. Both fault features and noise impulses exist in the raw signal (see Figure 2). Therefore, the local peak values involve faultrelated information and also reflect noise distribution with time. Consequently, for time $t$ in the signal, the temporal intervals between the two contiguous peaks are selected as the length of SE at that moment.

For a temporal signal of $N_{\mathrm{lp}}$ local peaks, $T_{k}$ denotes the moment where the $k$ th local maximum value appears and $A_{k}$ denotes the $k$ th time interval between the two relevant contiguous local peaks. They can be formulated as follows:

$$
\begin{aligned}
& T=\left\{T_{k} \mid k=1,2,3, \ldots, N_{\mathrm{lp}}\right\}, \\
& A=\left\{A_{k} \mid A_{k}=T_{k+1}-T_{k}, k=1,2,3, \ldots, N_{\mathrm{lp}}-1\right\} .
\end{aligned}
$$

Let $B(n)$ denote the local peak nearest to the $n$th discrete signal in set $T$.

$$
\begin{aligned}
B(n)=\max \left\{T_{n}^{\prime} \mid T_{n}^{\prime} \in T, T_{n}^{\prime} \leq n\right\} & \\
n & =0,1,2, \ldots, N-1 .
\end{aligned}
$$

Let $I(n)$ denote the index of $B(n)$ in set $T$. The length of the SE $g_{n}(m)$ for the $n$th discrete sample can be defined as follows:

$$
L\left(g_{n}\right)=A_{I(n)}, \quad n=0,1,2, \ldots, N-1
$$

3.2. Features Extraction. The difference between the morphological operator and a variable SE length is adopted to process the original signals to effectively suppress the noise and enhance the fault-related feature. Thereafter, nine features will be extracted from the enhanced signal: skewness, kurtosis, clearance factor, shape factor, crest factor, impulse indicator, variance, square root amplitude value, and absolute mean amplitude value. The following list shows the nine statistical features and with their formulations.

$$
\text { Kurtosis: }(1 / N) \sum_{i=1}^{N} s_{i}^{4}
$$




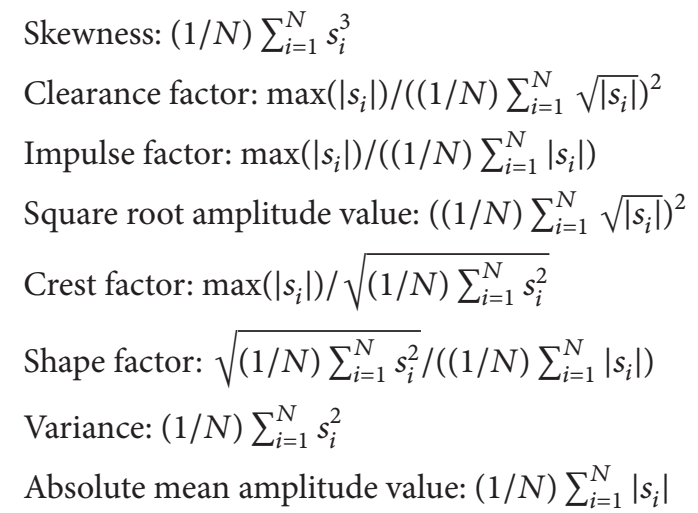

3.3. SVR Classification. After the utilization of morphological analysis and feature extraction, training sets $\left\{x_{i}, y_{i}\right\}_{i=1}^{L}$ are generated, where $x_{i}$ denotes the input feature vector, $y_{i}$ is the label that denotes the fault type, and $L$ is the number of samples. In practice, machinery fault type is constantly characterized by features in a nonlinear manner. SVR has been proven to exhibit immense potential in describing the nonlinear relationship between the input features and their output labels. Furthermore, for the samples under the same healthy state of the machinery, the extracted feature vectors are correlative to a certain extent. Thus, when these feature vectors are fed to the trained SVR model, their output values should be similar. From this point of view, a classification function based on the regression function in (10) can be derived. For a vector $x_{i}$ with an unknown label, $y_{i}$ can be achieved by using the following formula:

$$
y_{i}=\arg \min _{m=1,2, \ldots, M}\left|m-\left(\sum_{i=1}^{n}\left(\alpha_{i}-\alpha_{i}^{*}\right) K\left(x_{i}, X\right)+b\right)\right|,
$$

where $m$ denotes the label of $x_{i}$.

Kernel function and penalty factor $C$ are two key components of the SVR model. The radial basis function (RBF) kernel is introduced in this research to easily apply nonlinear mapping and rapidly optimize the parameters. RBF kernel is shown as follows:

$$
K\left(x_{i}, x\right)=\exp \left(\frac{-\left\|x_{i}-x\right\|^{2}}{2 \sigma^{2}}\right),
$$

where $\sigma$ is a positive real number and denotes the width parameter of the function. Hence, the optimization of $\sigma$ and $C$ is necessary to establish an accurate SVR model. The procedures for parameter optimization are shown in Figure 3. The search scope of $\sigma$ and search range of $C$ are 0.01 to 0.09 with step 0.01 and 1 to 32 with step 1 , respectively. Thereafter, various $\sigma$ and $C$ are selected in a linear order of the searching set. Meanwhile, the training samples are randomly divided into five groups, and the selected $\sigma$ and $C$ are used to establish the five training models. The average accuracy of the five models is defined as the accuracy of the SVR model. Lastly, when all $\sigma$ and $C$ had been evaluated, the accuracy of each SVR model is compared. Accordingly, the SVR model with the highest accuracy is used as the final model and its parameter would be the optimal $\sigma$ and $C$.

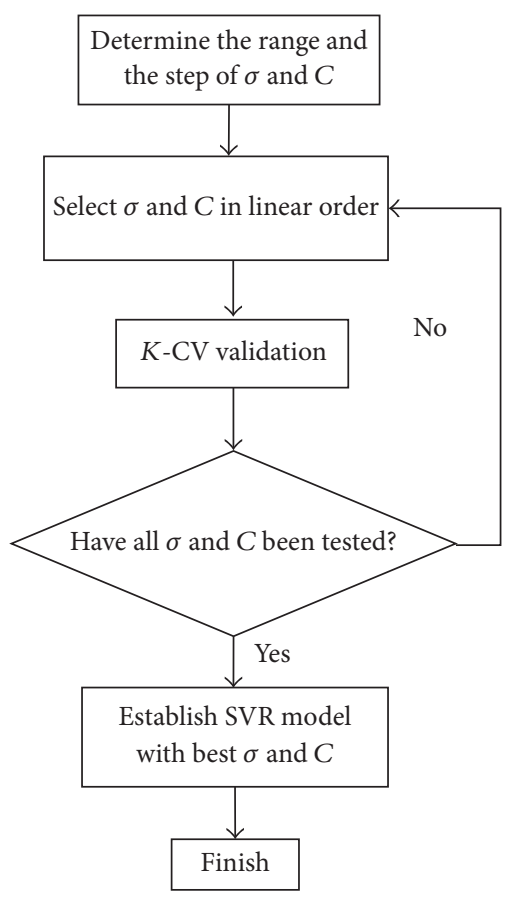

FIGURE 3: Flow diagram for the optimization of SVR model.

To avoid the states of overlearning and insufficient learning, increasing the credibility of classification result, $K$-fold cross-validation $(K-C V)[32,33]$ is adopted to train the optimal SVR model; that is, the training samples are divided into $K$ groups ( $K$ is often over 3; in this study, $K$ is set as $5)$. Each subset is used as a validation set, whereas the other $K-1$ groups are used as training sets. Hence, $K$ regression models exist, and the accuracy of the $K-\mathrm{CV}$ regression model is defined as the average performance of these $K$ regression models.

\section{Experimental Validations}

The bearing vibration signals supplied by the Case Western Reserve University are used to verify the effectiveness of the intelligent fault diagnosis scheme. The test rig comprises a $2 \mathrm{hp}$ motor, test bearing, and accelerometers (see Figure 4). The acceleration data was measured in the 12 oclock position of both the fan and drive ends. The digital data used in this analysis were collected at 12,000 samples per second. The horsepower and speed data were also collected using an encoder and torque transducer. The tested bearing is 6205-2RS JEM SKF and the detailed information is presented in Table 1. The data set used in this study was collected under four conditions: inner race fault, ball fault, outer race fault, and healthy state. A total of 58 signal samples were obtained under each state; 29 of these samples are used for training and the rest are used for testing. These four conditions are labeled as $1,2,3$, and 4 in sequence.

The original one-dimension signal is shown in Figure 5. The proposed varying-scale morphological analysis method is employed to process the raw signal. The local peaks 
TABLE 1: Geometry information of the test bearing.

\begin{tabular}{lcccc}
\hline $\begin{array}{l}\text { Outer race } \\
\text { diameter } \\
\text { (inch) }\end{array}$ & $\begin{array}{c}\text { Inner race } \\
\text { diameter } \\
\text { (inch) }\end{array}$ & $\begin{array}{c}\text { Pitch } \\
\text { diameter } \\
\text { (inch) }\end{array}$ & $\begin{array}{c}\text { Roller } \\
\text { diameter } \\
\text { (inch) }\end{array}$ & $\begin{array}{c}\text { The number } \\
\text { of rollers }\end{array}$ \\
\hline 2.0472 & 0.9843 & 1.537 & 0.3126 \\
\hline
\end{tabular}

TABLE 2: Diagnosis results of processed and raw signals.

\begin{tabular}{|c|c|c|c|c|c|}
\hline & Healthy & Inner race fault & Roller fault & Outer race fault & Overall \\
\hline \multicolumn{6}{|l|}{ Processed signal } \\
\hline Accuracy of training & $100 \%$ & $100 \%$ & $100 \%$ & $100 \%$ & $100 \%$ \\
\hline Accuracy of testing & $96.6 \%$ & $100 \%$ & $100 \%$ & $100 \%$ & $99.1 \%$ \\
\hline \multicolumn{6}{|l|}{ Raw signal } \\
\hline Accuracy of training & $100 \%$ & $100 \%$ & $100 \%$ & $100 \%$ & $100 \%$ \\
\hline Accuracy of testing & $100 \%$ & $100 \%$ & $89.65 \%$ & $100 \%$ & $97.41 \%$ \\
\hline
\end{tabular}

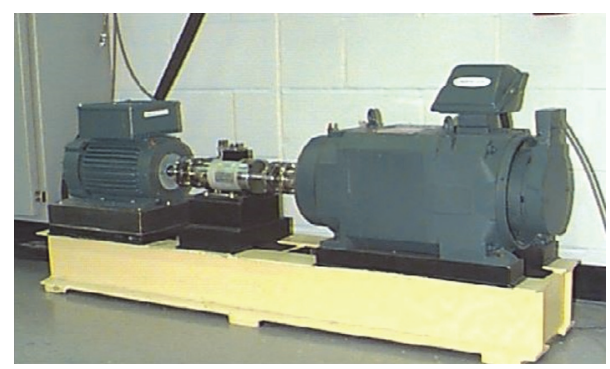

FIGURE 4: Experimental facility.

are determined first based on the procedures depicted in Section 3, and their time intervals are calculated thereafter. At various time points, the length of SE is defined dynamically, following ((12)-(14)). Lastly, the difference between the morphological operator and variable SE length is adopted to process the raw vibration signals to effectively suppress the noise and enhance the fault-related feature. The morphologically filtered signal is shown in Figure 6. Evidently, the processed signals have been well denoised with the aid of adaptive morphological analysis.

Statistical features are extracted from the filtered vibration signal thereafter. Kurtosis is known to measure the degree of steepness, and the variance value reflects the fluctuation to the mean value of the signal. The absolute mean amplitude and square root amplitude values are decided by the signal energy. The nine statistical features evidently focus considerable attention on various aspects of the machine fault signals. When various faults occur, the signal amplitude distribution, energy, and impact intervals will change prominently. Hence, the feature vector formed by the nine indicators can substantially present the healthy state of a machine due to the combination of sensitive features. Consequently, each sample is represented by a feature vector with nine elements after signal processing and feature extraction.

Thereafter, 29 feature vectors under each bearing fault pattern are extracted from the training set. A total of 116 training samples exist in four conditions. Thus, the size of the training and testing matrices is $116 \times 9$. $\mathrm{K}$-CV is performed during the training process. This strategy can avoid the states of overlearning and insufficient learning, as well as eventually increasing the classification credibility of the optimal SVR model. The training results are shown in Figure 7 . When the output of the model is below 1.5, the fault type is inner race fault; between 1.5 and 2.5 , the fault type is ball fault; between 2.5 and 3.5, the fault type is outer race fault; and over 3.5, the state is healthy. Thus, all samples shown in Figure 7 are correctly identified based on (15), thereby validating the optimal SVR model. The 29 testing samples under each bearing fault pattern are also preprocessed following the same procedure, and a total of 116 feature vectors are extracted. Thereafter, they are fed to the optimal SVR model and their fault patterns are derived using the proposed support vector regressive classification method.

The testing results are presented in Figure 8. Similarly, only one sample from the healthy state is misclassified to ball fault, whereas the other testing samples are classified properly. Table 2 summarizes the results in Figures 7-9. Upon following the proposed intelligent machinery fault diagnosis method, each step exhibits its satisfactory performance with a testing accuracy of up to $99.1 \%$. This result is higher than that obtained by the raw signal (Figure 9).

Furthermore, a comparative study as shown in Table 3 is conducted between the proposed method and those in [34, 35]. Vakharia et al. [34] proposed the permutation entropy to perform a proper wavelet analysis and conduct fault classification using the artificial neural network and SVM. Abbasion et al. [35] introduced a method that optimized the signal decomposition levels and recognized the bearing fault by using wavelet analysis and SVM, respectively. The misclassified sample may be a bad sample because the SVR model output showed a large error from its reference value. By contrast, the outputs of the other samples were within a small range away from their reference values. Although the testing accuracy achieved by the proposed method is slightly lower than $100 \%$, only one sample was misclassified (see the results shown in Figure 8). Moreover, the sample number is 58 for each state, whereas these values are 40 and 9 in [34] and [35], respectively. 


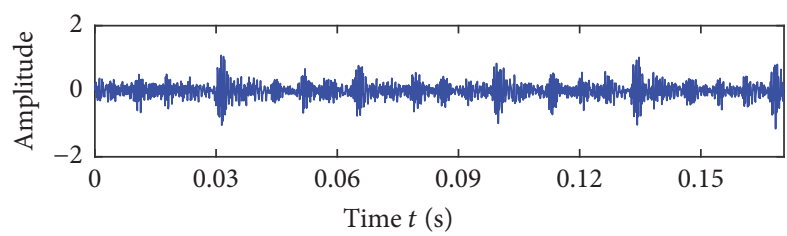

(a)

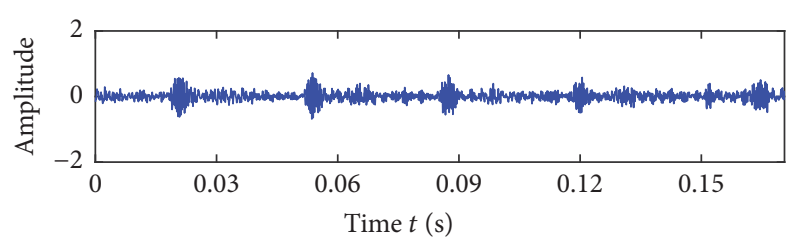

(c)

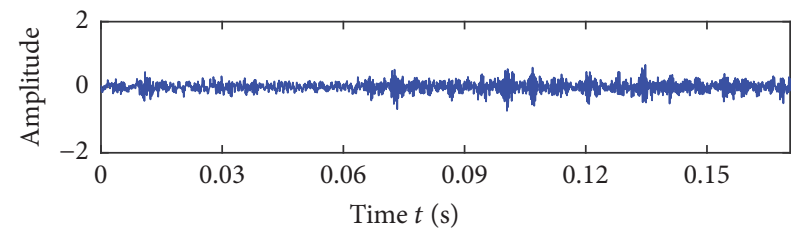

(b)

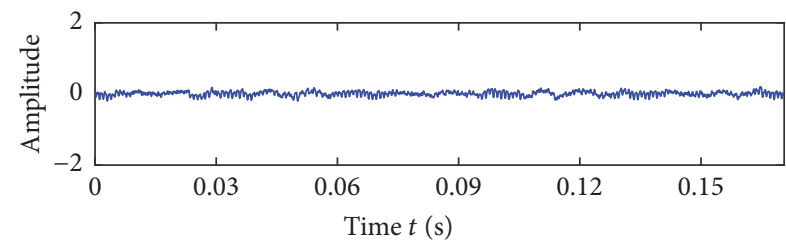

(d)

FIgURE 5: Raw vibration signal: (a) inner race fault, (b) ball fault, (c) outer race fault, and (d) healthy state.

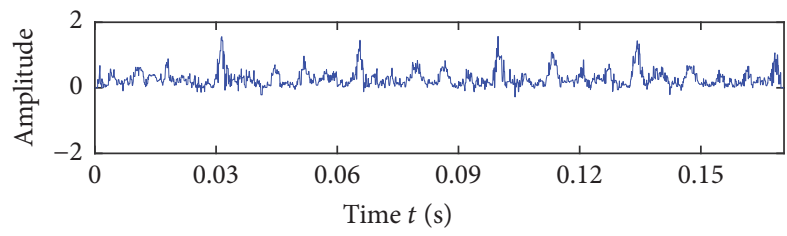

(a)

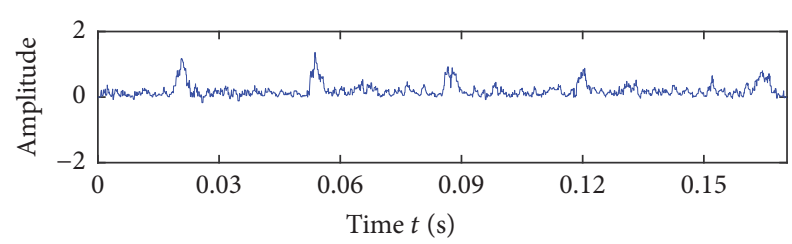

(c)

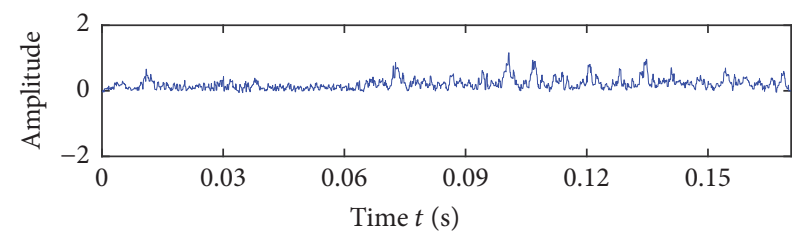

(b)

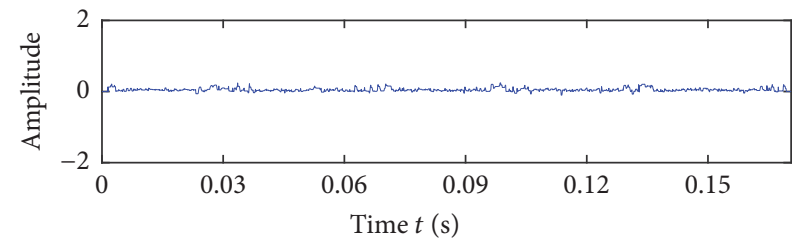

(d)

Figure 6: Processed signal via varying-scale morphological analysis: (a) inner race fault, (b) ball fault, (c) outer race fault, and (d) healthy state.

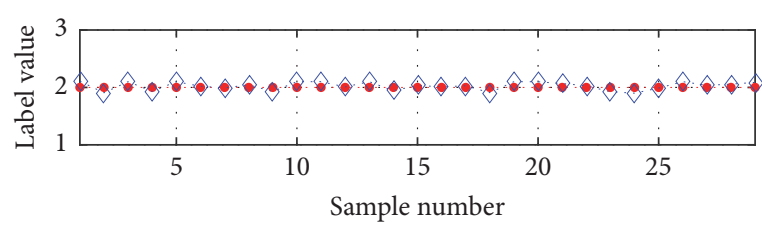

Actual sample label Model output value

(a)

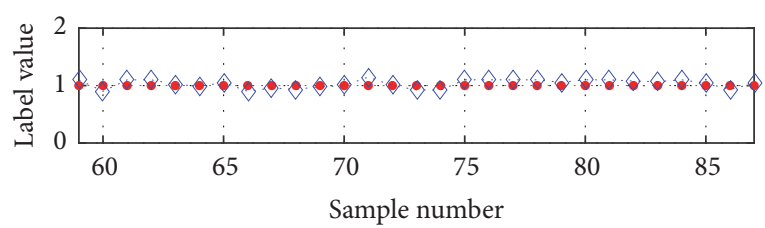

-. Actual sample label .. Model output value

(c)

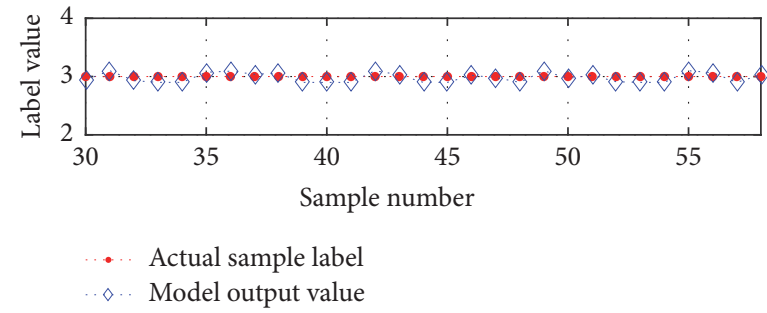

(b)

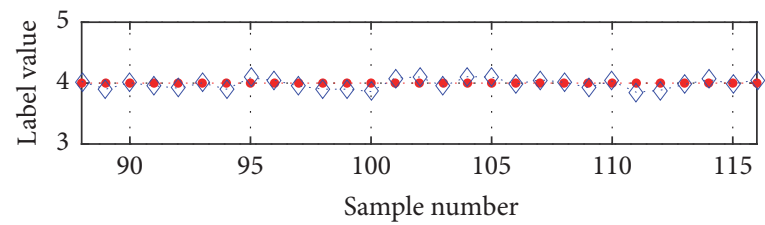

-.. Actual sample label $\diamond$.. Model output value

(d)

FIGURE 7: Results of the processed training samples. 
TABLE 3: Comparative study between the proposed method and the previous work.

\begin{tabular}{lcccc}
\hline Methods & Proposed method & ANN method & SVM method & SVM and wavelet denoising \\
\hline Accuracy of training & $100 \%$ & $97.5 \%$ & $97.5 \%$ & $100 \%$ \\
Accuracy of testing & $99.1 \%$ & $97.5 \%$ & $97.5 \%$ & $100 \%$ \\
\hline
\end{tabular}

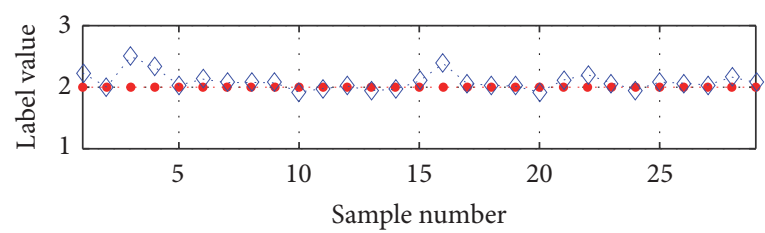

-. Actual sample label

$\diamond$. Model output value

(a)

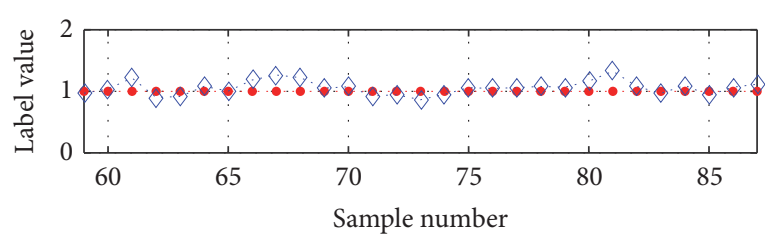

-.. Actual sample label

$\diamond$. Model output value

(c)

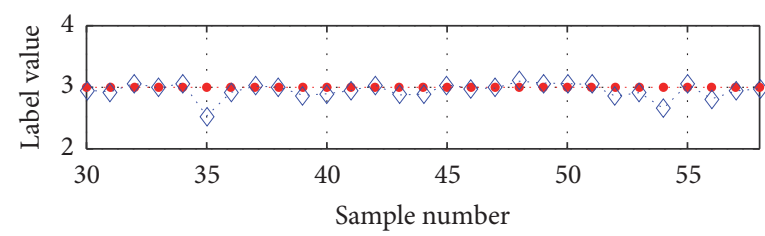

-.. Actual sample label

$\diamond$.. Model output value

(b)

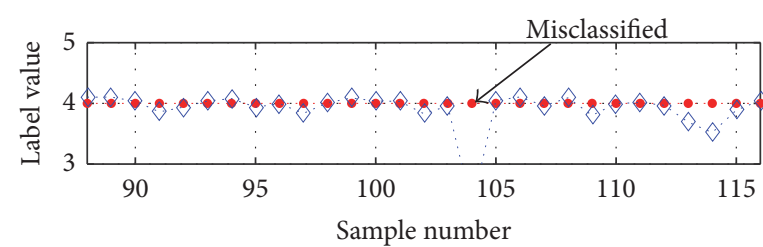

-. Actual sample label

$\diamond$.. Model output value

(d)

FIGURE 8: Results of the processed testing samples.

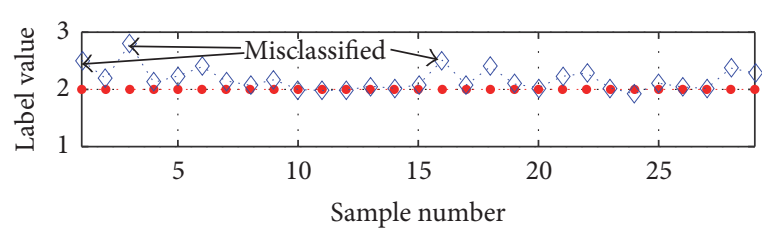

-. Actual sample label

$\diamond$. Model output value

(a)

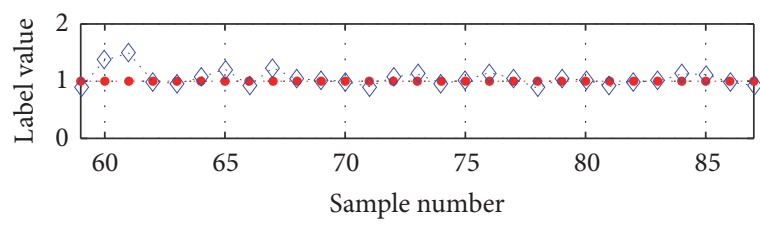

-. Actual sample label

$\diamond$. Model output value

(c)

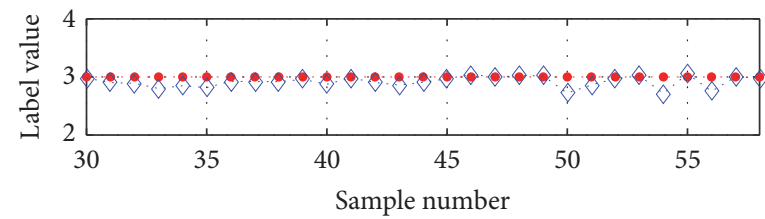

-.. Actual sample label

$\diamond$. Model output value

(b)

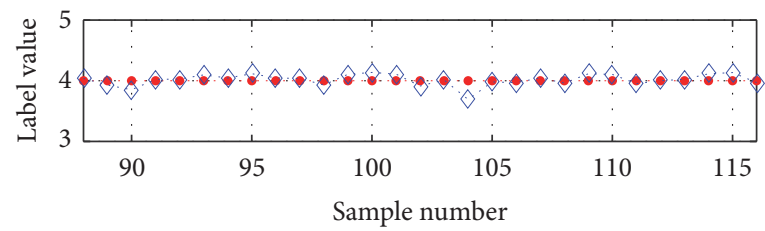

-.. Actual sample label

$\diamond$.. Model output value

(d)

FIGURE 9: Results of unprocessed testing samples.

\section{Conclusion}

This study presents a novel intelligent machinery fault diagnosis method. This method includes the extraction of morphological features and SVR classifier. Morphological analysis is a nonlinear method that can measure and extract the mechanical fault impact-related component from the vibration signals using SEs. The performance is subjected to the proper selection of SEs. First, an adaptive morphological analysis that dynamically varies the scales of SEs is proposed to effectively reduce the effect of noise and enhance the fault-related feature. Thereafter, nine features are extracted 
from the processed signal as feature vectors. SVR theory is used as basis to develop a regressive decision function to overcome the shortcomings of the traditional SVM method. The SVR model is optimized through $K-\mathrm{CV}$, and the optimal model is further used for testing. The results obtained in the investigation of the bearing data set show that the proposed scheme reaches a high degree of accuracy in machinery fault diagnosis and good generalizability compared with previous methods.

\section{Conflicts of Interest}

The authors declare that they have no conflicts of interest.

\section{Acknowledgments}

This research was financially supported by the National Natural Science Foundation of China (Grant nos. 51505311 and 51375322), the Natural Science Foundation of Jiangsu Province (no. BK20150339), and the China Postdoctoral Science Foundation funded project (2016T90490).

\section{References}

[1] D. Wang, W. Guo, and X. Wang, "A joint sparse wavelet coefficient extraction and adaptive noise reduction method in recovery of weak bearing fault features from a multi-component signal mixture," Applied Soft Computing Journal, vol. 13, no. 10, pp. 4097-4104, 2013.

[2] Y. Liu, B. He, F. Liu, S. Lu, and Y. Zhao, "Feature fusion using kernel joint approximate diagonalization of eigen-matrices for rolling bearing fault identification," Journal of Sound \& Vibration, vol. 385, pp. 389-401, 2016.

[3] Y. Lei, F. Jia, J. Lin, S. Xing, and S. X. Ding, "An intelligent fault diagnosis method using unsupervised feature learning towards mechanical big data," IEEE Transactions on Industrial Electronics, vol. 63, no. 5, pp. 3137-3147, 2016.

[4] J. Chen, Z. Li, J. Pan et al., "Wavelet transform based on inner product in fault diagnosis of rotating machinery: a review," Mechanical Systems and Signal Processing, vol. 70-71, pp. 1-35, 2016.

[5] J. Ben Ali, N. Fnaiech, L. Saidi, B. Chebel-Morello, and F. Fnaiech, "Application of empirical mode decomposition and artificial neural network for automatic bearing fault diagnosis based on vibration signals," Applied Acoustics, vol. 89, pp. 16-27, 2015.

[6] J. Seshadrinath, B. Singh, and B. K. Panigrahi, "Investigation of vibration signatures for multiple fault diagnosis in variable frequency drives using complex wavelets," IEEE Transactions on Power Electronics, vol. 29, no. 2, pp. 936-945, 2014.

[7] F. Cong, W. Zhong, S. Tong, N. Tang, and J. Chen, "Research of singular value decomposition based on slip matrix for rolling bearing fault diagnosis," Journal of Sound and Vibration, vol. 344, pp. 447-463, 2015.

[8] R. Tiwari, V. K. Gupta, and P. K. Kankar, "Bearing fault diagnosis based on multi-scale permutation entropy and adaptive neuro fuzzy classifier," JVC/Journal of Vibration and Control, vol. 21, no. 3, pp. 461-467, 2015.

[9] D. Wang, P. W. Tse, and K. L. Tsui, "An enhanced Kurtogram method for fault diagnosis of rolling element bearings,"
Mechanical Systems and Signal Processing, vol. 35, no. 1-2, pp. 176-199, 2013.

[10] Y. Zhang, B. Tang, and X. Xiao, "Time-frequency interpretation of multi-frequency signal from rotating machinery using an improved Hilbert-Huang transform," Measurement, vol. 82, pp. 221-239, 2016.

[11] P. Maragos and R. W. Schafer, "Morphological filters-Part I: their set-theoretic analysis and relations to linear shiftinvariant filters," Institute of Electrical and Electronics Engineers. Transactions on Acoustics, Speech, and Signal Processing, vol. 35, no. 8, pp. 1153-1169, 1987.

[12] N. Lin and A. Dufresne, "Surface chemistry, morphological analysis and properties of cellulose nanocrystals with gradiented sulfation degrees," Nanoscale, vol. 6, no. 10, pp. 5384-5393, 2014.

[13] S. Dong, L. Chen, B. Tang, X. Xu, Z. Gao, and J. Liu, "Rotating machine fault diagnosis based on optimal morphological filter and local tangent space alignment," Shock and Vibration, vol. 2015, Article ID 893504, 9 pages, 2015.

[14] S. Rajabi, M. Samanazari, H. R. Momeni, and A. Ramezani, "Automated fault diagnosis of rolling element bearings based on morphological operators and M-ANFIS," in Proceedings of the 24th Iranian Conference on Electrical Engineering (ICEE '16), pp. 1757-1762, IEEE, Shiraz, Iran, May 2016.

[15] Y. Li, M. J. Zuo, J. Lin, and J. Liu, "Fault detection method for railway wheel flat using an adaptive multiscale morphological filter," Mechanical Systems and Signal Processing, vol. 84, pp. 642-658, 2017.

[16] S. Hong, S. Fang-Jian, C. Bo, and Q. Wei, "Research of gear fault detection in morphological wavelet domain," Journal of Physics: Conference Series, vol. 679, no. 1, Article ID 012035, 2016.

[17] A. S. Raj and N. Murali, "Early classification of bearing faults using morphological operators and fuzzy inference," IEEE Transactions on Industrial Electronics, vol. 60, no. 2, pp. 567-574, 2013.

[18] H. Li, Y. Wang, B. Wang, J. Sun, and Y. Li, “The application of a general mathematical morphological particle as a novel indicator for the performance degradation assessment of a bearing," Mechanical Systems and Signal Processing, vol. 82, no. 1, pp. 490-502, 2014.

[19] D. Yu, M. Wang, and X. Cheng, "A method for the compound fault diagnosis of gearboxes based on morphological component analysis," Measurement, vol. 91, pp. 519-531, 2016.

[20] V. Bhateja, S. Urooj, R. Mehrotra, R. Verma, A. Lay-Ekuakille, and V. D. Verma, "A composite wavelets and morphology approach for ECG noise filtering," in Pattern Recognition and Machine Intelligence, vol. 8251 of Lecture Notes in Computer Science, pp. 361-366, Springer, Berlin, Germany, 2013.

[21] M.-L. Zhang and Z.-H. Zhou, "A review on multi-label learning algorithms," IEEE Transactions on Knowledge and Data Engineering, vol. 26, no. 8, pp. 1819-1837, 2014.

[22] V. Vapnik, The Nature of Statistical Learning Theory, Springer Science \& Business Media, Berlin, Germany, 2013.

[23] A. Widodo and B.-S. Yang, "Support vector machine in machine condition monitoring and fault diagnosis," Mechanical Systems and Signal Processing, vol. 21, no. 6, pp. 2560-2574, 2007.

[24] B. Gu, V. S. Sheng, Z. Wang, D. Ho, S. Osman, and S. Li, "Incremental learning for $\mathcal{v}$-Support Vector Regression," Neural Networks, vol. 67, pp. 140-150, 2015.

[25] A. Kazem, E. Sharifi, F. K. Hussain, M. Saberi, and O. K. Hussain, "Support vector regression with chaos-based firefly algorithm 
for stock market price forecasting," Applied Soft Computing Journal, vol. 13, no. 2, pp. 947-958, 2013.

[26] A. Kavousi-Fard, H. Samet, and F. Marzbani, "A new hybrid Modified Firefly Algorithm and Support Vector Regression model for accurate Short Term Load Forecasting," Expert Systems with Applications, vol. 41, no. 13, pp. 6047-6056, 2014.

[27] Z. Wei, T. Tao, D. ZhuoShu, and E. Zio, "A dynamic particle filter-support vector regression method for reliability prediction," Reliability Engineering and System Safety, vol. 119, pp. 109116, 2013.

[28] K. Chen and J. Yu, "Short-term wind speed prediction using an unscented Kalman filter based state-space support vector regression approach," Applied Energy, vol. 113, pp. 690-705, 2014.

[29] J. Serra, Image Analysis and Mathematical Morphology, vol. 1, Academic Press, Cambridge, Mass, USA, 1982.

[30] L. Zhang, D. Yang, J. Xu, and Z. Chen, "Approach to extracting gear fault feature based on mathematical morphological filtering," Jixie Gongcheng Xuebao, vol. 43, no. 2, pp. 71-75, 2007.

[31] A. J. Smola and B. Schölkopf, "A tutorial on support vector regression," Statistics and Computing, vol. 14, no. 3, pp. 199-222, 2004.

[32] T.-T. Wong, "Performance evaluation of classification algorithms by k-fold and leave-one-out cross validation," Pattern Recognition, vol. 48, no. 9, pp. 2839-2846, 2015.

[33] K. J. Grimm, G. L. Mazza, and P. Davoudzadeh, "Model selection in finite mixture models: a $k$-fold cross-validation approach," Structural Equation Modeling: A Multidisciplinary Journal, vol. 24, no. 2, pp. 246-256, 2016.

[34] V. Vakharia, V. K. Gupta, and P. K. Kankar, "A multiscale permutation entropy based approach to select wavelet for fault diagnosis of ball bearings," Journal of Vibration \& Control, vol. 21, no. 16, pp. 3123-3131, 2015.

[35] S. Abbasion, A. Rafsanjani, A. Farshidianfar, and N. Irani, "Rolling element bearings multi-fault classification based on the wavelet denoising and support vector machine," Mechanical Systems \& Signal Processing, vol. 21, no. 7, pp. 2933-2945, 2007. 


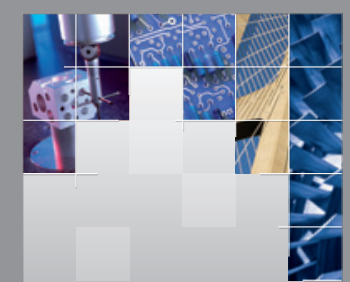

\section{Enfincering}
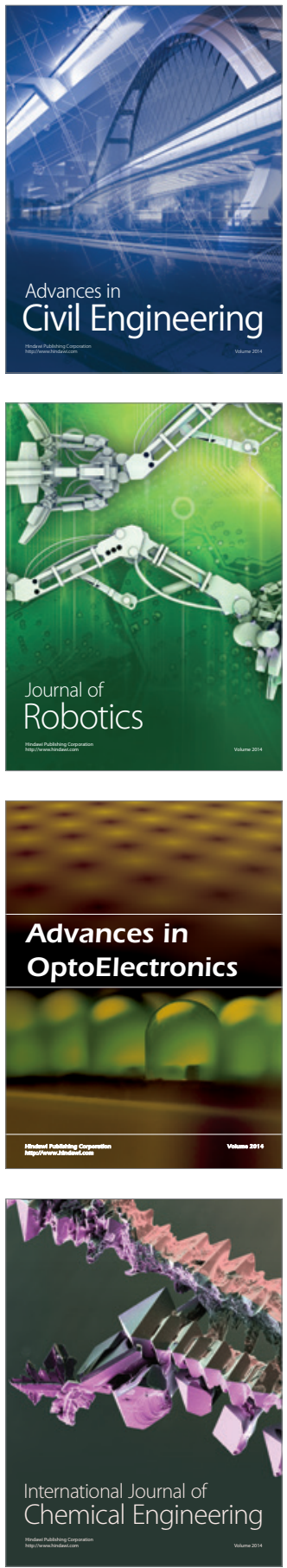

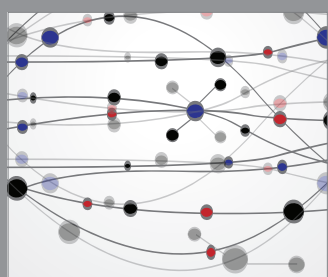

The Scientific World Journal

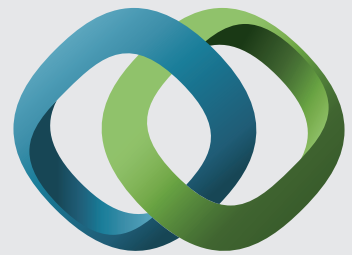

\section{Hindawi}

Submit your manuscripts at

https://www.hindawi.com
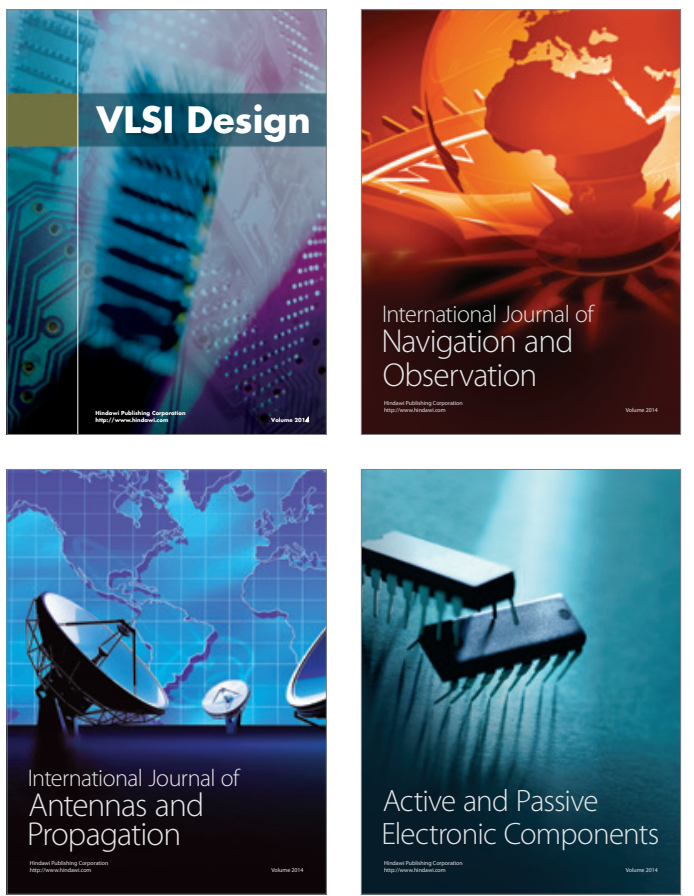
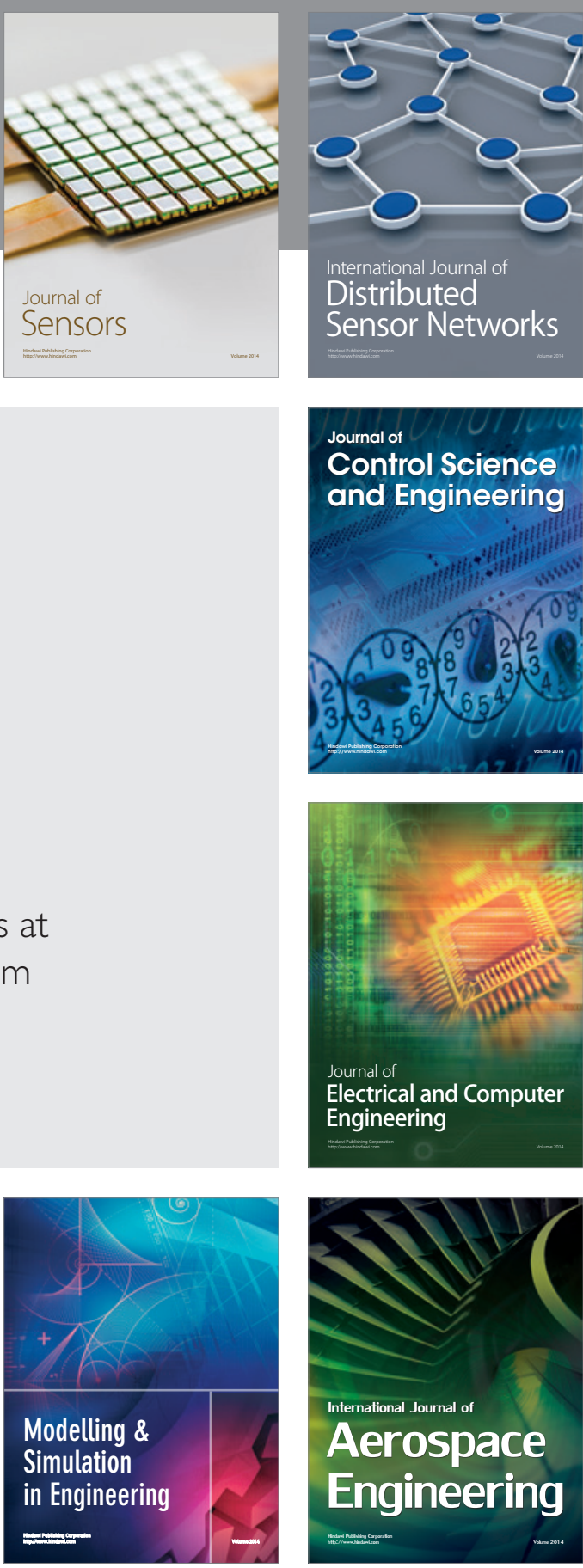

International Journal of

Distributed

Sensor Networks

$-$

Joumal of

Control Science

and Engineering
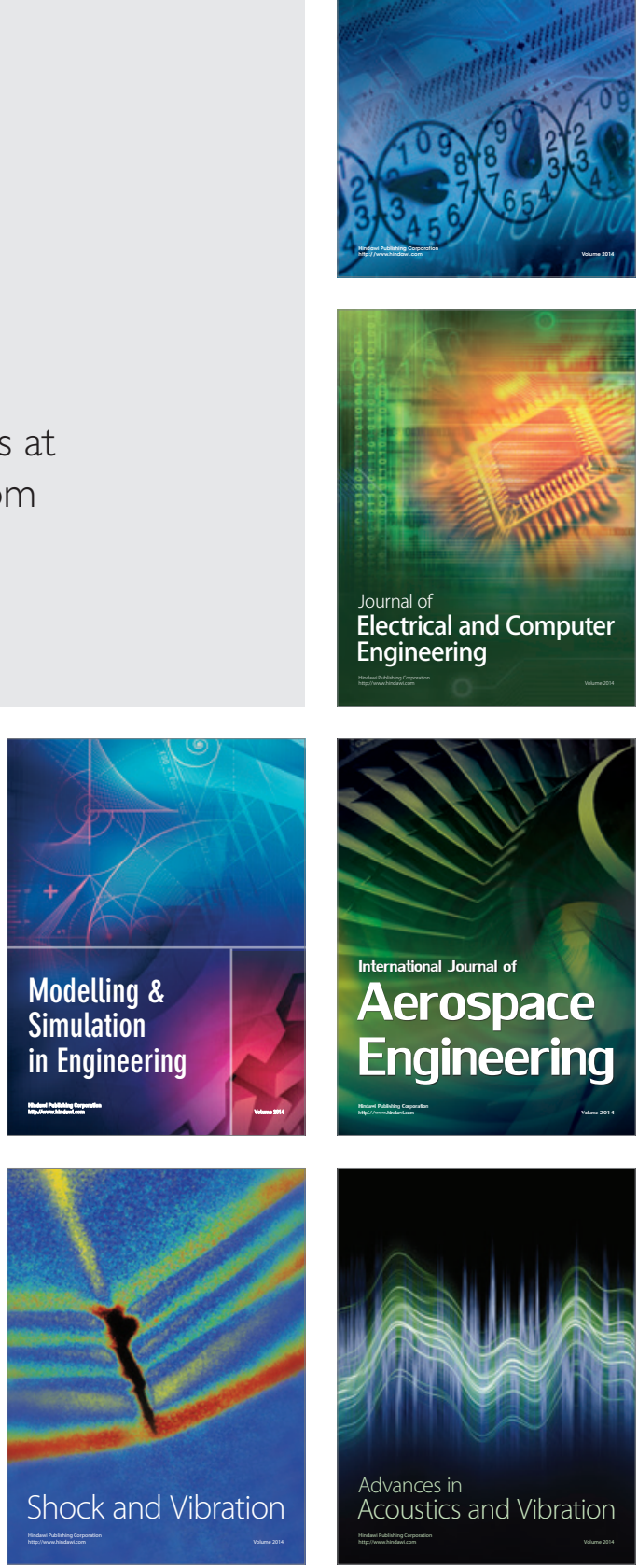